\title{
Las inyecciones intra-articulares de solución salina tienen similar eficacia a las de plasma rico en plaquetas, ácido hialurónico o corticoides para el alivio del dolor en pacientes con artrosis de cadera: meta-análisis en red
}

\author{
Intra-articular saline solution injections have similar efficacy to those of platelet-rich plasma, hyaluronic acid or \\ corticosteroids for pain relief in patients with hip osteoarthritis: network meta-analysis
}

\section{Comentado de:}

Gazemdam A, et al. Br J Sports Med. 2020 Aug 22;bjsports2020-102179. PMID: 32829298'.

\section{Objetivo}

Evaluar la eficacia comparativa de varios tratamientos de inyecciones intra-articulares (IA) en pacientes con artrosis de cadera respecto del dolor y la funcionalidad.

\section{Diseño y fuente de datos}

Revisión sistemática y meta-análisis en red realizado a partir de una busqueda en PUBMED, Embase, SCOPUS, el Registro Cochrane de Ensayos Clínicos Controlados, y la Web of Science, de investigaciones publicadas hasta octubre de 2019.

\section{Selección de estudios y extracción de datos}

Fueron incluidos estudios controlados y aleatorizados que habían comparado la eficacia de inyecciones intra-articulares de plasma rico en plaquetas (PRP), corticoides (C), ácido hialurónico $(\mathrm{AH})$ y placebo (solución salina: SS), con un seguimiento de hasta seis meses. Fueron extraídas las características de los pacientes y su lugar de reclutamiento, el tipo de cegamiento y el financiamiento, así como los puntajes de dolor y funcionalidad empleados.

Se estableció como la mínima diferencia clínicamente relevante a una reducción de 1,3 puntos en la escala visual analógica de dolor (0 a 10), y de 7,1 puntos en la escala de WOMAC de funcionalidad de la cadera (de 0 a 68).

\section{Resultados Principales}

Fueron incluidos 11 estudios que habían reclutado un total de 1.353 pacientes con una edad promedio de $64 \pm 9,5$ años (54\% mujeres). 314 participantes habían recibido inyecciones IA de placebo (SS), 596 con AH, 237 con C, 155 con PRP, 31 con AH más PRP y 20 pacientes no recibieron IA.

Excepto los pacientes que recibieron PRP más $A H$, en todas las ramas evaluadas se observó una mejoría del dolor y de la funcionalidad. Sin embargo, ninguna intervención fue más eficaz que la inyección IA de placebo para el alivio del dolor ni para mejorar resultados funcionales a ninguno de los horizontes temporales evaluados (dos a cuatro meses, o seis meses). No se observaron diferencias de eficacia vinculadas a si el ensayo clínico había sido o no patrocinado por la industria farmacéutica.

Fuente de financiamiento/Conflicto de interés de los autores: La fuente de financiamiento del estudio no fue declarada por los autores. Uno de ellos comunicó haber recibido honorarios de AgNovos Healthcare, Sanofi Aventis, Smith \& Nephew y Stryker, así como subsidios de investigación por parte de DJ Orthopedics y Ferring Pharmaceuticals, en el contexto de otras investigaciones diferentes a la resumida en este artículo.

\section{Comentario}

Los resultados de este meta-análisis en red abren el interrogante sobre el significado de lo que implica punzar una articulación, independientemente de lo que inyectemos en ella. Es conocido que al realizar una inyección intra-articular se produce activación plaquetaria y de factores de crecimiento, así como cambios inflamatorios que provocan un beneficio en la zona tratada ${ }^{2}$, más allá efecto psicológico de saber que se está recibiendo un tratamiento para una afección.

Por otro lado, hace varios años que se propone abandonar a las inyecciones de solución salina como placebo, ya que en múltiples estudios ha demostrado un efecto terapéutico durante por lo menos seis meses luego de la inyección ${ }^{3}$. Estudios experimentales han documentado que las soluciones hiperosmolares como la solución salina y la dextrosa hipertónica protegen a los condrocitos de las lesiones mecánicas ${ }^{4}$ y bloquean los receptores TRPV1, favoreciendo la reparación del tejido. Nuestro grupo de trabajo tiene particular experiencia con el uso de dextrosa hipertónica, con resultados similares a los documentados en este meta-análisis en red ${ }^{5,6}$ respecto de la solución salina.

\section{Conclusiones del comentador}

Dado que por el momento, podemos concluir que no existe evidencia de que alguno de los tratamientos basados en inyecciones intra-articulares sea superior al resto, para aliviar el dolor y para mejorar la funcionalidad de los pacientes con artrosis de cadera, las decisiones terapéuticas tendrán que estar basadas en el intersticio que existe entre la mejor experiencia del equipo tratante y los valores y las creencias del paciente $y / 0$ su entorno familiar. 


\section{Referencias}

1. Gazendam A, Ekhtiari S, Bozzo A, et al. Intra-articular saline injection as effective as corticosteroids, platelet-rich plasma and hyaluronic acid for hip osteoarthritis pain: a systematic review and network meta-analysis of randomised controlled trials. Br J Sports Med. 2020;p. bjsports-2020-102179. Available from: 10.1136/bjsports-2020-102179.

2. Reeves KD, Sit RW, Rabago DP. Dextrose prolotherapy: a narrative review of basic science, clinical research and treatment recommendations. Phys Med Rehabil Clin N Am. 2016;27(4):783-823. Available from: 10.1016/j.pmr.2016.06.001.

3. Saltzman BM, Leroux T, Meyer MA, et al. The Therapeutic Effect of Intra-articular Normal Saline Injections for Knee Osteoarthritis: A Meta-analysis of Evidence Level 1 Studies. Am J Sports Med. 2017;45(11):2647-2653. Available from: 10.1177/0363546516680607;https://dx.doi.org/10.1177/ 0363546516680607.

4. Amin A, Huntley JS, Patton JT, et al. Hyperosmolarity protects chondrocytes from mechanical injury in human articular cartilage: an experimental report. J Bone Joint Surg Br. 2011;93(2):277-84. Available from: 10.1302/0301-620X.93B2.24977.

5. Sit RW, Wu RWK, Rabago D, et al. Efficacy of intraarticular Hypertonic Dextrose (prolotherapy) for knee osteoarthritis: a randomized controlled trial. Ann Fam Med. 2020;18(3):235-242. Available from: 10.1370/afm.2520.

6. Gül D, Orsçelik A, Akpancar S. Treatment of osteoarthritis secondary to development dysplasia of the hip with prolotherapy injection vs a supervised progressive exercise control. Med Sci Monit. 2020;26:919166-919166. Available from: 10.12659/MSM.919166. 